tinuclear antibodies and rheumatoid factor, were negative, and levels of angiotensin-converting enzyme were negative. Tests for antibodies against the human immunodeficiency virus (HIV) and for HIV p24 antigen were negative, and the erythrocyte sedimentation rate was $53 \mathrm{~mm}$ per hour. The patient was treated intravenously with phenytoin and high-dose penicillin G for 21 days. Magnetic resonance imaging of the brain six days after the initiation of therapy showed no enhancement of the left frontal lesion, and one month later the abnormal signal had disappeared (Fig. 1B). Studies of cerebrospinal fluid six months after the completion of therapy were normal, with a nonreactive VDRL test.

The findings of abnormalities on magnetic resonance imaging and a reactive VDRL test of cerebrospinal fluid, along with a therapeutic response to intravenous penicillin, made the diagnosis of neurosyphilis almost certain in our patient. ${ }^{2}$ The incidence of neurosyphilis has increased over the past two decades, particularly in immunocompromised hosts. ${ }^{3,4}$ In patients presenting with focal motor status epilepticus and corresponding abnormally enhancing lesions on magnetic resonance imaging of the brain, the diagnosis of neurosyphilis should be seriously considered regardless of their immune status. The response to treatment should be evaluated by follow-up radiologic and cerebrospinal fluid studies.

Jose I. SUAREZ, M.D. DiANE MLAKAR, R.N. Susan M. SNOdgrass, M.D. University Hospitals of Cleveland Cleveland, $\mathrm{OH} 44106$

1. Hooshmand H. Seizure disorders associated with neurosyphilis. Dis Nerv Syst 1976;37:133-6

2. Hook EW III. Diagnosing neurosyphilis. Clin Infect Dis 1994;18:295

3. Simon RP. Neurosyphilis. Neurology 1994;44:2228-30.

4. Musher DM, Baughn RE. Neurosyphilis in HIV-infected persons. N Engl J Med 1994;331:1516-7.

\section{Antiplatelet and Anticoagulant Therapy after Coronary-Artery Stenting}

To the Editor: Schömig et al. (April 25 issue) ${ }^{1}$ report that they did not observe neutropenia in any of the $257 \mathrm{pa}^{-}$ tients receiving short-term ticlopidine therapy after coronary stenting. The authors note that in the literature, all reported cases of neutropenia were fully reversible and had developed in the second and third month of long-term therapy with ticlopidine. For these reasons, Schömig et al. consider the possible side effect of neutropenia with shortterm ticlopidine therapy to be of little clinical importance. We do not agree with this view because of our clinical experience and other reports in the Journal ${ }^{2}$ not mentioned by Schömig et al.

We observed severe neutropenia ( 84 neutrophils per cubic millimeter) in a 79-year-old man 23 days after the initiation of ticlopidine therapy because of coronary-artery stenting. Blood counts had been performed every third day after stenting, and the total white-cell count declined on the 20th day.

The medical literature reports that agranulocytosis may occur as early as 15 or 27 days after the start of ticlopidine therapy, may not always be reversible, and in rare cases may be fatal. ${ }^{2}$ The manufacturer recommends blood-count monitoring every other week for the first three months of ticlopidine therapy. In their study, Schömig et al. performed blood counts in their patients only during the first 10 days. Thus, most of their patients received ticlopidine over an ensuing period of at least 18 days without blood counts. Apparently, this approach caused no harm to their patients. However, we suggest that during short-term ticlopidine therapy, blood counts be performed at least every other week or if symptoms develop - particularly with signs of infection.

Arno A. Schöneberger, M.D. Kurt SCHMidT, M.D.

Krankenversorgung der Bundesbahnbeamten Hospital 61462 Königstein, Germany

1. Schömig A, Neumann F-J, Kastrati A, et al. A randomized comparison of antiplatelet and anticoagulant therapy after the placement of coronaryartery stents. N Engl J Med 1996;334:1084-9.

2. Prevention of ischemic stroke. N Engl J Med 1995;333:460.

To the Editor: Schömig et al. conclude that "as compared with conventional anticoagulant therapy, combined antiplatelet therapy after the placement of coronary-artery stents reduces the incidence of both cardiac events and hemorrhagic and vascular complications." Some aspects of the study need to be clarified before these conclusions are generally applied to patients who undergo coronary-artery stenting.

The main indications for stenting in the study were "extensive coronary-artery dissection after PTCA [percutaneous transluminal coronary angioplasty], complete vessel closure, [and] residual stenosis of 30 percent or more." These conditions are associated with an increased risk of acute or subacute stent thrombosis. ${ }^{1}$ The authors state that "all patients in whom stenting was successful (i.e., in whom the stent was placed at the desired position and there was less than 30 percent residual stenosis)" were eligible for randomization. Excluded from the study were "patients in whom stenting was intended primarily as a bridge to aortocoronary bypass grafting, who had cardiogenic shock, or who had needed mechanical ventilation before undergoing PTCA." The number of patients excluded was very high. A total of 517 patients were eligible and consented to be randomized, and 109 patients were excluded (17.4 percent). Also, the mean percentage of residual stenosis after stenting in the enrolled patients was less than 3 percent, a value indicating the successful placement of stents in the enrolled patients. These data suggest that only patients with excellent results after coronary stenting were included, and a substantial number of patients with a high risk of thrombotic occlusion of the stent were excluded before randomization. It would be interesting to know the specific causes of exclusion and the in-hospital course of the excluded patients.

In addition, the authors report the dissection rate before coronary stenting but not the incidence of residual dissection after placement of the stent. How many patients 
had coronary dissection after stenting in the two randomized groups? Residual dissection after intracoronary-stent implantation is an important predictor of subacute stent thrombosis. ${ }^{1}$ Different outcomes in the two randomized groups could be explained by different rates of residual dissection after stent placement.

ANgel CeQuier, M.D.
MANEl SAbaté, M.D.
Enric Esplugas, M.D.
Hospital de Bellvitge
08907 Barcelona, Spain

1. Mak KH, Belli G, Ellis SG, Moliterno DJ. Subacute stent thrombosis: evolving issues and current concepts. J Am Coll Cardiol 1996;27:494503

The authors reply:

To the Editor: Schöneberger and Schmidt and Cequier and colleagues raise important questions about the generalizability of our findings to the large number of patients who undergo coronary-stent implantation. Cequier and colleagues are concerned that we might have preferentially excluded high-risk patients. Among the 626 consecutive patients undergoing stent implantation on our service during the study period, the most frequent reason for ineligibility was the patient's refusal to participate in the study, which accounted for 9.9 percent of the patients. Other reasons for ineligibility were procedural failure in 3.7 percent of the patients, cardiogenic shock or the need for mechanical ventilation due to acute myocardial infarction before the intervention in 2.7 percent, an absolute indication for anticoagulation therapy in 0.6 percent, and the use of a stent as a bridge to bypass grafting in 0.5 percent. In none of the patients was residual dissection after stenting a reason for exclusion. The frequency of a major residual dissection after stenting was 1.5 percent in the entire study group, and the frequency did not differ significantly between the two treatment groups.

We do not consider our patient population a low-risk cohort, because 59 percent of the target lesions were classified as type C. Moreover, 24 percent of our patients presented with acute myocardial infarction, and 45 percent with unstable angina. Subgroup analysis shows that patients at high risk for stent occlusion, such as those with acute myocardial infarction, ${ }^{1}$ benefit the most from combined antiplatelet therapy.

Drs. Schöneberger and Schmidt point out that severe neutropenia can develop as soon as 15 days after treatment with ticlopidine. However, at such an early stage, neutropenia appears to be exceedingly rare. In 1529 patients who took part in the largest trial of ticlopidine, ${ }^{2}$ neutropenia did not occur during the first month. Blood-count monitoring after discharge from the hospital was not part of our study protocol and was left to the discretion of the patients' physicians, who carried on with the treatment after discharge. We believe that blood counts should be performed during the third and fourth week of treatment. Most important, continuation of ticlopidine therapy after four weeks should be strictly avoided. With such surveillance, the risk of neutropenia can be minimized and thus should not limit the administration of combined antiplatelet therapy after coronary-stent placement.

FranZ-Josef Neumann, M.D. Hanna Walter, M.D. Albert SCHÖMIG, M.D.

Technische Universität München 81675 Munich, Germany

1. Walter H, Neumann F-J, Richardt G, et al. Antiplatelet vs. anticoagulation treatment after intracoronary Palmaz-Schatz stent placement in acute myocardial infarction - a prospective randomized trial. J Am Coll Cardiol 1996;27:Suppl A:279A. abstract.

2. Hass WK, Easton JD, Adams HP Jr, et al. A randomized trial comparing ticlopidine hydrochloride with aspirin for the prevention of stroke in highrisk patients. N Engl J Med 1989;321:501-7.

\section{Aspirin and Coronary Disease}

To the Editor: In his editorial on platelets and coronary artery disease (April 25 issue), ${ }_{1}^{1}$ Handin states, "Aspirin has now become an essential cardiovascular drug, and it is unusual to find patients with established coronary artery disease who do not take it." Sadly, despite overwhelming evidence of the clinical benefit of aspirin in patients with coronary heart disease, many if not most patients with established disease do not take aspirin. Only 37 percent of patients received aspirin in the Scandinavian Simvastatin Survival Study. ${ }^{2}$ Reviewing the treatment of Medicare patients with acute myocardial infarction, the Cooperative Cardiovascular Project found that only 50 percent of patients received aspirin within 48 hours after the diagnosis of infarction. ${ }^{3}$ Moreover, the mortality rate at six months among patients not receiving aspirin was twice the rate among those receiving it. ${ }^{4}$ Studying community practice, Shahar et al. found a low level of aspirin use among patients with symptomatic coronary heart disease: 58 percent among white men, 35 percent among black men, 34 percent among white women, and 13 percent among black women..$^{5}$ Physicians' misperceptions of the available data are a substantial problem. Ayanian et al. found that only 55 percent of internists believe that aspirin definitely improves the long-term prognosis after myocardial infarction. ${ }^{6}$

Documentation of similar deficiencies in care abounds in medicine. The reasons for not prescribing effective therapies are complex and may involve cost considerations. In the case of aspirin, high cost is not a factor.

Robert A. Vogel, M.D

University of Maryland School of Medicine Baltimore, MD 21201-1595

1. Handin RI. Platelets and coronary artery disease. N Engl J Med 1996 ; 334:1126-8.

2. Scandinavian Simvastatin Survival Study Group. Randomised trial of cholesterol lowering in 4444 patients with coronary heart disease: the Scandinavian Simvastatin Survival Study (4S). Lancet 1994;344:1383-9.

3. Ellerbeck EF, Jencks SF, Radford MJ, et al. Quality of care for Medicare patients with acute myocardial infarction: a four-state pilot study from the Cooperative Cardiovascular Project. JAMA 1995;273:1509-14.

4. Krumholz HM, Radford MJ, Ellerbeck EF, et al. Aspirin for secondary prevention after acute myocardial infarction in the elderly: prescribed use and outcomes. Arch Intern Med 1996;124:292-8.

5. Shahar E, Folsom AR, Romm FJ, et al. Patterns of aspirin use in middle- 